\title{
An acousto-electromagnetic sensor for locating land mines
}

\author{
Waymond R. Scott, Jr. ${ }^{\mathrm{a}}$, Chistoph Schroeder ${ }^{\mathrm{a}}$ and James S. Martin ${ }^{\mathrm{b}}$ \\ ${ }^{a}$ School of Electrical and Computer Engineering \\ ${ }^{\mathrm{b}} \mathrm{School}$ of Mechanical Engineering \\ Georgia Institute of Technology \\ Atlanta, GA 30332
}

\begin{abstract}
A hybrid technique is presented that simultaneously uses both electromagnetic and acoustic waves in a synergistic manner to detect buried land mines. The system consists of an electromagnetic radar and an acoustic source. The acoustic source causes both the mine and the surface of the earth to be displaced. The electromagnetic radar is used to detect these displacements and, thus, the mine. To demonstrate the viability of this technique, an experimental system has been constructed. The system uses an electrodynamic transducer to induce an acoustic surface wave, a tank filled with damp sand to simulate the earth, a simulated mine, and a radar to measure the vibrations. The technique looks promising; we have been able to detect both simulated antipersonnel mines and antitank mines buried in damp sand from the experimental results obtained with the system.
\end{abstract}

Keywords: land mine, mine detection, acoustic, ground penetrating radar, GPR

\section{INTRODUCTION}

A technique to detect land mines that simultaneously uses both electromagnetic and acoustic waves in a synergistic manner is currently being investigated. The synergism has the potential to significantly enhance the signature of the mine with respect to the clutter and make it possible to detect a mine that would be impossible to detect by purely electromagnetic or purely acoustic means.

The configuration of the system currently being studied is shown in figure 1. The system consists of an electromagnetic radar and an acoustic source. The acoustic source induces an acoustic (seismic) wave into the earth. The acoustic wave causes both the mine and the surface of the earth to be displaced. The displacement of the mine is different than the earth, because the acoustic properties of the mine are quite different than those of the earth. The displacement of the surface of the earth when a mine is present is different than when it is not present because of the waves scattered from the mine. The electromagnetic radar is used to detect these displacements and, thus, the mine. This idea has been discussed previously [1,2], but it has not been seriously investigated.

In addition, the radar can be simultaneously used in a mode in which only the electromagnetic waves are used to identify (image) the mine. In this electromagnetic only mode, the radar is a conventional ground penetrating radar (GPR). Thus, both the acoustic and electromagnetic properties of the mine can be used to differentiate it from the earth. It may be possible to locate or identify mines with the GPR that are invisible to the acoustic system or visa versa. Or it may me possible to combine the output of both the GPR and the acoustic system to lower the false alarm rate of the system. For example, a wet packed soil is generally very lossy for electromagnetic waves; thus, it is difficult to find the mines using the electromagnetic only mode. However, these soils tend to be lower loss for acoustic waves; thus, the acousto-electromagnetic mode may perform well in these soils. The opposite is also true. A loose dry soil is generally very lossy for acoustic waves; thus, it will be difficult to find the mine in this soil using the acousto-electromagnetic mode. However these soils tend to be low loss for electromagnetic waves; thus, the electromagnetic only mode may perform well in these soils. 
An experimental model for the system has been constructed and automated. The experimental model is being used to demonstrate the viability of the technique and to study the interactions of the acoustic and electromagnetic waves with buried mines. The technique looks promising; we have been able to detect both simulated antipersonnel mines and antitank mines buried in damp sand. However, additional investigation of the technique is needed to determine the capabilities of the technique in more varied conditions. A two-dimensional finitedifference time-domain (FDTD) model for the acoustic waves has been also developed and is being used to help understand the interactions of the acoustic waves and the mines.

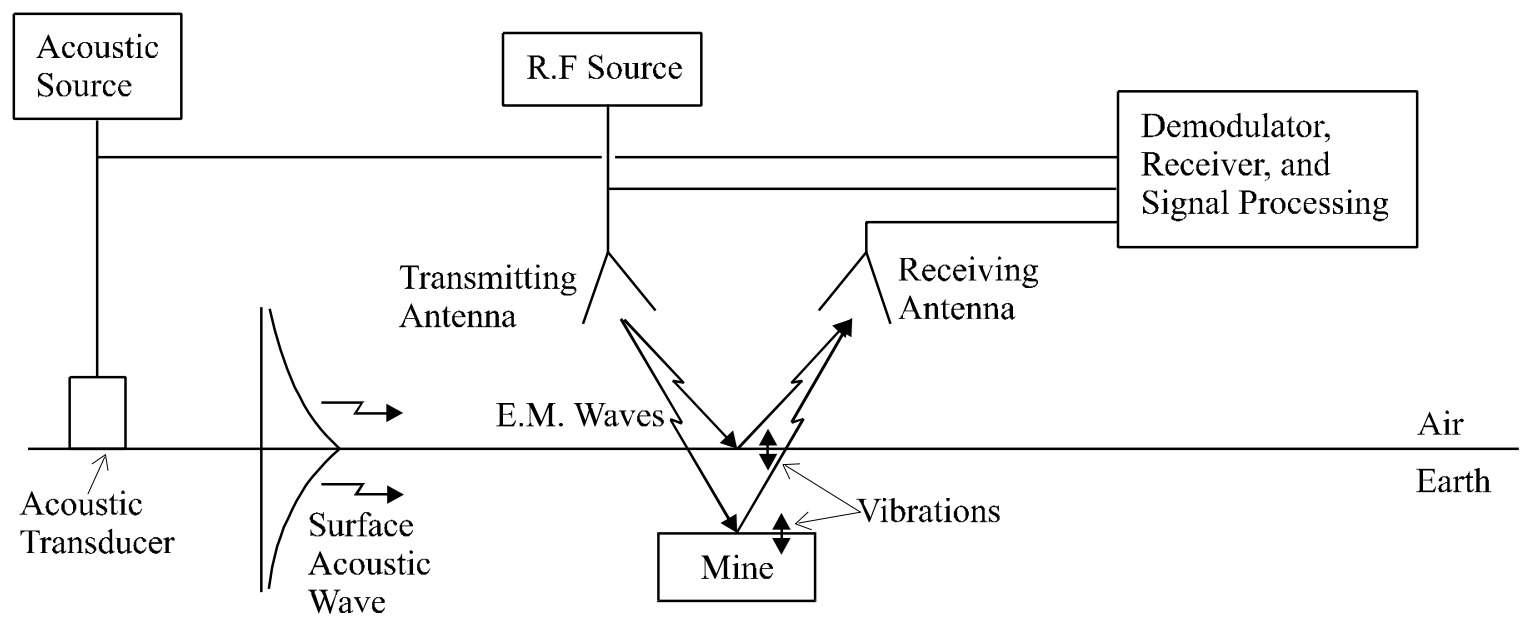

Figure 1. Acousto-electromagnetic mine detection system with the acoustic transducer placed on the surface of the earth.

\section{EXPERIMENTAL MODEL}

A radar has been designed and built to measure the displacements of the surface of the earth and the mine. Figure 2 is a schematic diagram of the radar. The radar radiates electromagnetic waves that are reflected off of a vibrating interface. The reflected waves are received by the radar, and a Homodyne system is used to demodulate the signals. The vibrations are determined from these demodulated signals. The two biggest challenges to make this radar perform adequately for the mine detection system are 1) to make it sufficiently sensitive to be able to detect the small vibrations, and 2) to make the spot size (the area on the surface illuminated by the electromagnetic waves) sufficiently small. The radar can measure vibrations as small as $1 \mathrm{~nm}\left(10^{-9} \mathrm{~m}\right)$ as currently configured. To obtain this sensitivity, the radar was designed to minimize the effects of noise, such as the phase noise of the source and the electromagnetic interference from low-frequency magnetic fields. The spot size of the radar must be smaller than approximately one half of a wavelength of the acoustic waves. Currently, a small spot size is obtained by using an open-ended waveguide as the antenna for the radar. This antenna produces a sufficiently small spot size when the open end of the antenna is placed within a few centimeters of the surface. This antenna is adequate for the experimental model, but probably will not be adequate for a fieldable system. We have investigated antennas that may be appropriate for a fieldable system, but we have not tried to incorporate one into the current radar. The radar can be configured in either a monostatic mode as in figure 2 or in a bistatic mode as in figure 1. Currently the radar is being used in the monostatic mode to get a smaller spot size, but the radar has been used and has performed well in the bistatic mode. The radar can be operated at frequencies between $2 \mathrm{GHz}$ and $8 \mathrm{GHz}$; however, all of the results presented in this paper are obtained with the radar operating at $8 \mathrm{GHz}$. The vibration of the surface of the sand has been measured with both the radar and an accelerometer; these measurements were compared and found to be in good agreement.

A drawing of the top view of the experimental model is shown in figure 3. The model consists of a tank that is filled with damp sand that has been packed to a relatively uniform density. The tank is approximately $120 \mathrm{~cm}$ 
wide, $120 \mathrm{~cm}$ deep, and $240 \mathrm{~cm}$ long. A transducer is placed on the surface of the sand and is used to launch the acoustic waves into the sand. The transducer is an electrodynamic shaker that is driven with a signal generator. The transducer has been coupled to the surface of the sand so that it preferentially launches acoustic surface waves. These surface waves travel across the surface of the tank and interact with a mine that is buried in the sand. The electromagnetic radar is used to measure the displacements caused by the acoustic waves. A x-y positioner is used to scan the radar over the surface of the sand. This system is under computer control, so it can scan the radar over the surface and record the data automatically.

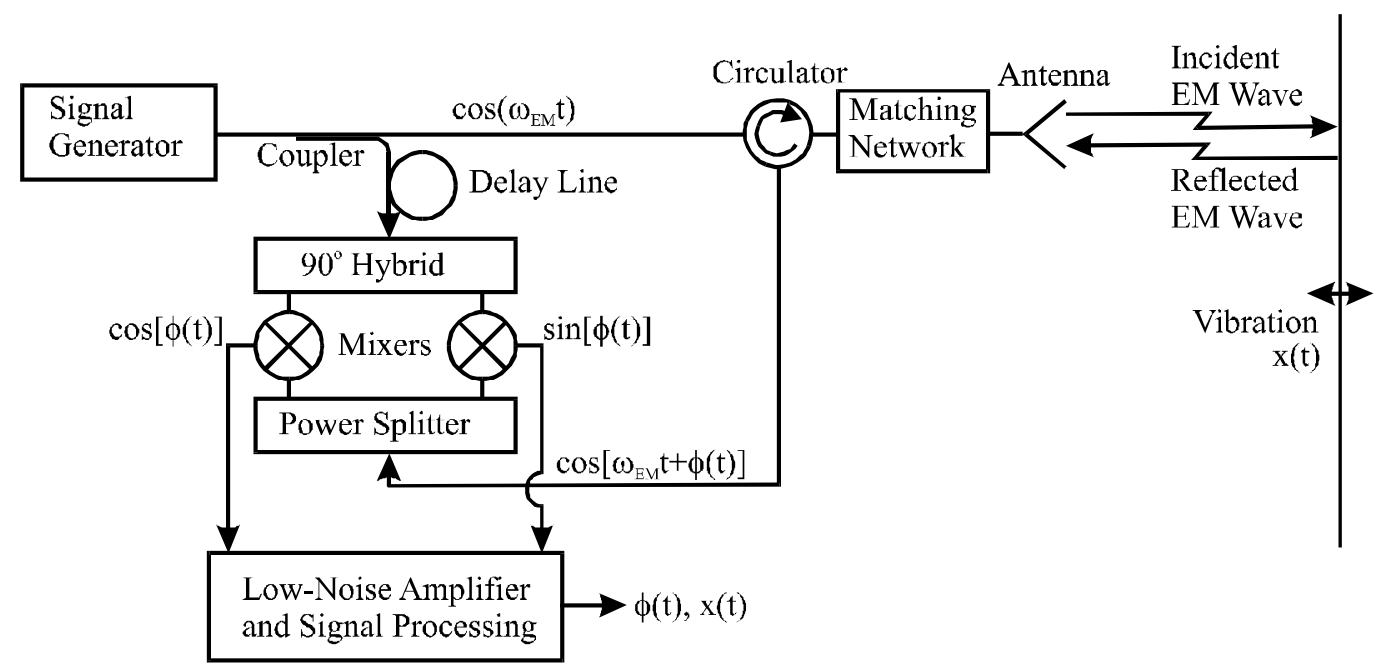

Figure 2 Schematic diagram of the radar

The region scanned with the radar is indicated on figure 3. The displacements are measured in this region as a function of time and position. The measurements are made on a uniform rectangular grid of discrete positions in the scanned region. The grid consists of 41 points in the $y$-direction that are spaced $2 \mathrm{~cm}$ apart and 121 points in the x-direction that are spaced $1 \mathrm{~cm}$ apart, for a total of 4961 points. The displacements were measured and recorded as a function of time at each of these points. Each of these time traces is averaged 256 times to reduce the noise. This noise is primarily due to background vibrations in the building that houses the model. The pulse repetition rate of the acoustic source must be significantly lower in the experimental setup than in the field, because of reverberation of the acoustic waves in the tank. It currently takes 24 to 48 hours to perform a complete scan; this is because the measurement is setup to obtain the maximum data integrity without concern for the scan time. The scan time can be greatly reduced by reducing the number of averages, increasing the pulse repetition rate, increasing the distance between sample positions on the surface, or arraying the radar so that multiple

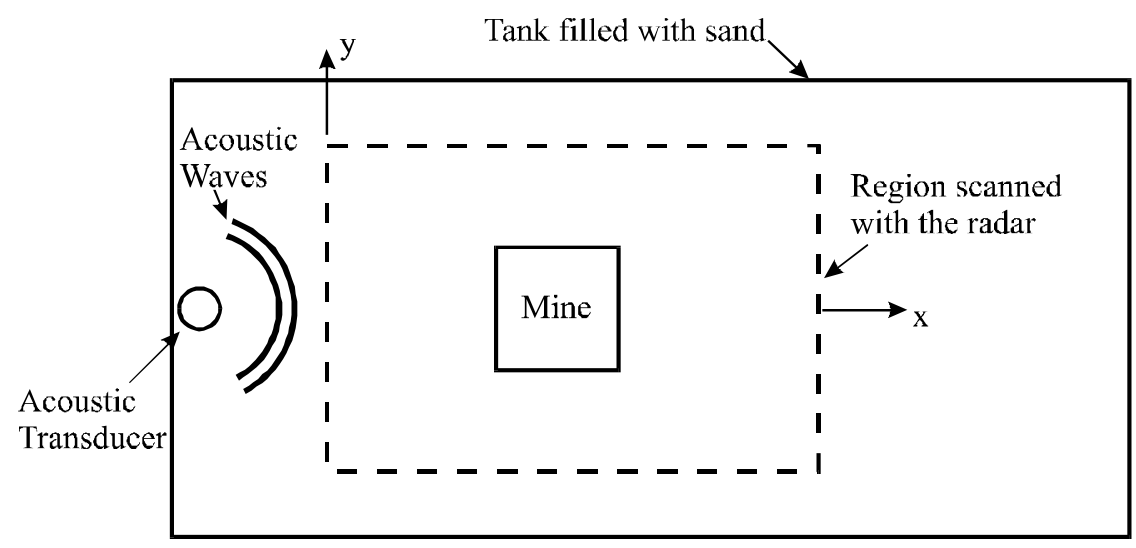

Figure 3 Top view of experimental model. 
positions can be measured simultaneously. For example, if the number of averages is reduced by a factor of 8 , the pulse repetition rate is increased by a factor of 5, the spacing between the points is increased by a factor of 2 in each direction, and the radar was arrayed to take 10 measurement points simultaneously; it would take approximately 1 to 2 minutes to perform a complete scan instead of 24 to 48 hours.

\section{EXPERIMENTAL RESULTS}

To date, the model has been used to study the interaction of the acoustic waves with two different mines: a simulated anti-tank mine made out of Acrylic plastic that is $30 \mathrm{~cm}$ wide, $30 \mathrm{~cm}$ long, and $7.5 \mathrm{~cm}$ high, and a simulated anti-personnel mine that is $7.6 \mathrm{~cm}$ in diameter and $2.5 \mathrm{~cm}$ high (the EM-3 inert mine). The anti-tank mine has been studied with it buried at three depths, and the anti-personnel mine has been studied at one depth. In these studies, the acoustic transducer is excited with a differentiated gaussian pulse. The pulse has a center frequency of $400 \mathrm{~Hz}$ for the anti-tank mine and a center frequency of $800 \mathrm{~Hz}$ for the anti-personnel mine.

Pseudo color graphs of the amplitude of the displacement of the surface are presented in figures 4 and 5 for four times. These results are for a simulated antitank mine buried in the sand. The top of the mine is flush with the surface of the sand for the graphs in figure 4, and the top of the mine is $5 \mathrm{~cm}$ below the surface for the graphs in figure 5. The position of the mine is indicated by the dotted white line. In these graphs, the color scale goes from black to blue to green to yellow to red to white. The smallest displacements are in black and the largest displacements are in white. At time \#1, the acoustic wave is seen traveling toward the mine. At times \#2 and \#3, the wave has reached the mine, and a portion of the wave is going across the mine while the rest is going around the mine. Notice that the portion of the wave that is going across the mine is ahead of the portion that is around the mine. This is because the mine is much stiffer than is the sand; thus, the wave appears to travel faster across the mine. The mine actually moves as if it is a rigid body. The motion of the mine is essentially a rocking motion excited by the wave motion in the sand. At time \#4, the wave is seen to be still going around the mine; however, a significant portion has passed across the mine. Notice that the wave that went across the mine is still ahead of the wave that went around the mine, and notice that the wave that went across the mine is smaller in amplitude than the one that went around the mine. Also notice that the displacements are smaller above the mine. The waves that are reflected from the mine can also be seen. The location and the shape of the mine are clearly evident in these graphs. We have also generated movies from the data recorded with the radar. In the movies, pseudo color graphs like those in figures 4 and 5 are stored and played back sequentially. The interaction of the waves with the mine is clearer in the movies than it is in figures 4 or 5; thus, it is easier to see the mine.

A waterfall graph of the displacement of the surface is presented in figure 6 for the anti-tank mine when the top of the mine is flush with the surface. In this graph, the displacement is plotted as a function of time for the 121 points spaced along the $\mathrm{x}$-axis with $\mathrm{y}=0$ (along the center of the scanned region). The plot for each of these 121 points is shifted vertically from the previous one. The plot for $x=0$ is at the bottom of the graph and the plot for $\mathrm{x}=120 \mathrm{~cm}$ is at the top of the graph. The region in which the mine is located is indicated in gray. The incident surface wave is seen to propagate toward the mine and to be reflected from the mine. The incident surface wave is also seen to travel across the mine. Notice that the wave travels faster across the mine; this is expected because the mine is stiffer than the sand. After the wave passes across the mine it is transmitted into the sand on the other side of the mine. Notice that the wave slows back down when it enters the sand. The incident pressure wave is also seen on the graph; however it is much smaller in amplitude than the surface wave. It is seen to propagate faster then the surface wave. The ripples on the top right of the graph are due to reflections of the incident wave off of the bottom of the tank. Note that the incident surface wave is spread out in time and more complex than the differentiated gaussian pulse that is the excitation for the transducer. This is partially due to the manner in which the wave is injected into the sand. We are investigating methods for injecting a more time-limited pulse.

When the top of the anti-tank mine is $10 \mathrm{~cm}$ below the surface, its effects can be seen in the recorded data; however, they are smaller and less obvious than when the mine is closer to the surface. This is because of the exponential decay in amplitude of the surface waves with depth. The rate of the exponential decay increases with increasing frequency; thus, only the lower frequency waves penetrate all the way down to the mine. The recorded 
vibrations are high-pass filtered at $100 \mathrm{~Hz}$; therefore, the frequencies that are the most sensitive to the mine have been removed. This is done because the tank that holds the sand has several strong resonances below $100 \mathrm{~Hz}$ that corrupt the measurements. These resonances will not occur when the measurement is made in the field; so it will be possible to use lower frequencies. Then the response of mine will be more obvious.

In an effort to enhance the signature of the mine, a simple signal-processing scheme was tried in which the forward traveling waves are filtered out leaving only the reflected waves. The reflected waves occur due to the interactions of the waves with a discontinuity in the sand such as a mine. Pseudo color graphs of the amplitude of the displacement of the surface of the sand are presented in figure 7 with the forward traveling waves filtered out. The top of the anti-tank mine is $10 \mathrm{~cm}$ below the surface. For time \#1, the incident pulse has just reached the mine and the reflected pulse is forming. For time \#2, the incident pulse has reached the mine and the reflected pulse is clearly seen. ${ }^{1}$ Thus, by filtering out the forward traveling waves, the response of the mine is clearly visible even when it is buried $10 \mathrm{~cm}$ deep. Measurements have not been made with the mine buried deeper to date, but we are planing to make such measurements.

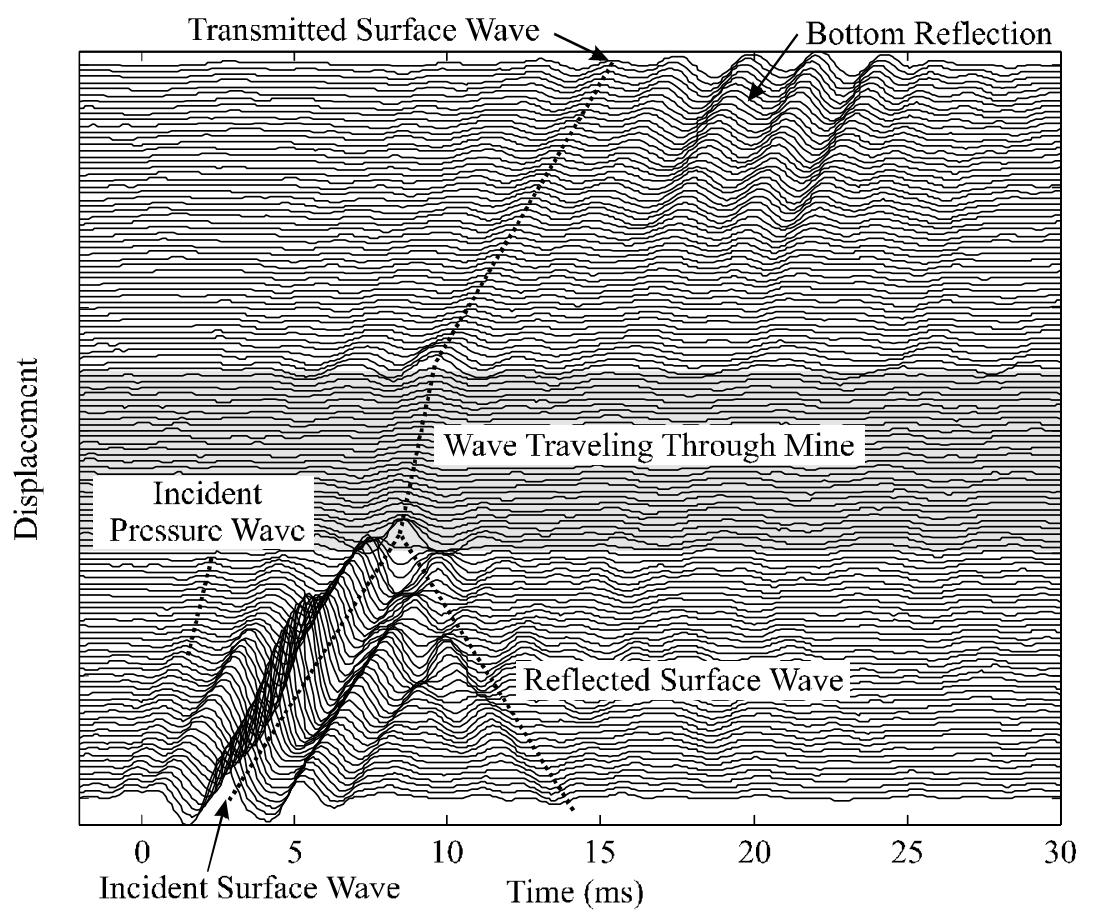

Figure 6 Waterfall graph of the displacement of the surface of the sand when the anti-tank mine is buried in the sand with $y=0$. The top of the mine is flush with the surface.

Pseudo color graphs of the amplitude of the displacement of the surface are presented in figure 7 for the antipersonnel mine. The forward traveling waves are filtered out, and the top of the mine is approximately $3 \mathrm{~mm}$ below the surface. For time \#1, the incident pulse has just reached the mine and a reflected pulse is seen being formed. For time \#2, the incident pulse has reached the mine and the reflected pulses are clearly seen. The effects of the anti-personnel mine can be clearly seen with the forward waves filtered out. The effects of the mine can be seen without the forward waves being filtered out, but the effects are smaller and less obvious. The effects of the anti-personnel mine would be stronger if a higher frequency incident pulse were used. So far we have been unable to get higher frequency pulses to propagate in the sand, because the sand is very lossy at these frequencies.

\footnotetext{
${ }^{1}$ Some of the smaller displacements seen in figure 7 are not due to reflections from the mine. They are an artifact of the method used to record the data. They are due to reflections off of the walls of the tank from previous incident pulses that have not died out. The measurement procedure has been modified to remove these artifacts.
} 
We believe that the higher frequencies will propagate better in a soil that contains clay, because the clay will bind the particles together. We plan to investigate materials other than sand to model the soil.

\section{NUMERICAL MODEL}

A two-dimensional FDTD model for the acoustic waves has been developed. Figure 8 is a diagram of the FDTD model. The waves are injected with a point source, a perfectly matched layer (PML) is used to absorb the waves at the edge of the mesh, and a free surface boundary condition is used on the boundary between the soil and the air. The results from the numerical model are in fairly good agreement with those from the experimental model. The numerical model has been very useful in helping us to understand the interaction of the acoustic wave with the mines. With the numerical model, the waves can be observed below the surface; whereas, with the experimental model, the waves can only be observed on the surface. For example, when the anti-tank mine is buried $10 \mathrm{~cm}$ deep; the lower-frequency surface waves can be seen to interact with and be reflected by the mine, while the higher frequency surface waves can be seen to propagate relatively unaffected over the mine. A surface wave can be converted into a pressure wave and visa versa, when the waves interact with the mine. The conversions are more evident in the numerical model than in the experimental model. This is because of the greater signal to noise ratio and the much lower reverberations (reflections from the walls) of the numerical model.

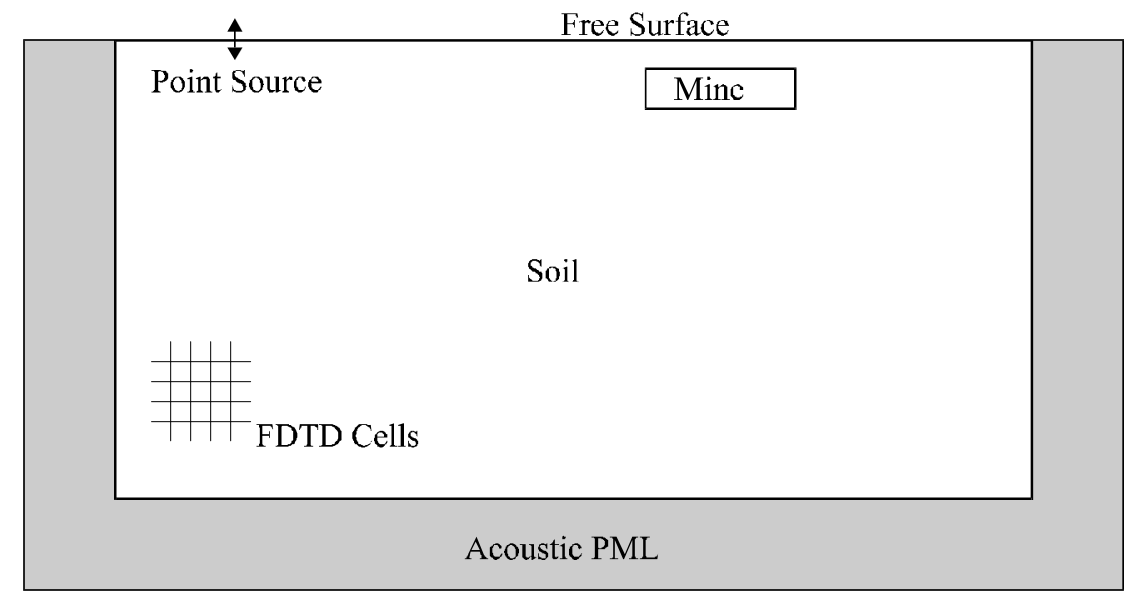

Figure 8 Diagram of the acoustic FDTD model.

Pseudo color graphs of the amplitude of the velocity of the particles in the soil are presented in figure 9 for the anti-tank mine buried $5 \mathrm{~cm}$ deep. The incident pulse is a differentiated gaussian pulse with a center frequency of $400 \mathrm{~Hz}$. The soil models the sand that is used in the experimental model. At time \#1, pressure (P), shear (S), and surface $(\mathrm{R})$ waves are seen to be launched. The shear and surface waves overlap, because they propagate at approximately the same velocity (the surface wave propagates slightly slower than the shear wave). The surface wave is the more intense wave near the surface. The pressure wave is ahead of the surface/shear wave because it propagates faster. At time \#2, the surface/shear wave is seen to have reached the mine. The portion of the wave that passed across the mine is seen to be ahead of the portion that is passing around the mine. Again this is because the wave travels faster in the mine than in the sand. Scattered pressure waves that are due to the interaction of the incident surface/shear wave with the mine are also seen. At time \#3, the incident wave is seen to have almost passed by the mine. Scattered pressure, shear, and surface waves are seen propagating away from the mine. At time \#4, the wave has passed by the mine. The scattered pressure, shear, and surface waves are still seen propagating away from the mine. Notice that a surface wave appears to be trapped above the mine, we have observed similar behavior in some of the experimental results. 
The numerical model predicts a stronger response for the anti-personnel mine than what is obtained with the experimental model. We believe that this difference is due to the numerical model being two dimensional, whereas the experimental model is three dimensional. We are planning to extend the numerical model to three dimensions.

\section{CONCLUSTIONS}

The acousto-electromagnetic sensor has been investigated using both numerical and experimental modeling. The technique looks promising; we have been able to detect both simulated antipersonnel mines and antitank

mines buried in damp sand from the experimental results. However, much more work is needed to understand the capabilities of the sensor in more varied conditions.

\section{ACKNOWLEDGEMENTS}

This work is supported in part by the US Army Research Office under contract DAAH04-96-1-0048.

\section{REFERENCES}

[1] G.S. Smith, "Summary Report: Workshop on New Directions for Electromagnetic Detection of Non-Metallic Mines,” Report for U.S. Army BRDEC and ARO, June 1992

[2] C. Stewart, Summary of Mine Detection Research, Vol. I, pp. 172-179, Tech. Report 1636-TR, May 1960, U.S. Army Engineering Res. and Devel. Labs, Corps. of Eng., Belvoir, VA. 

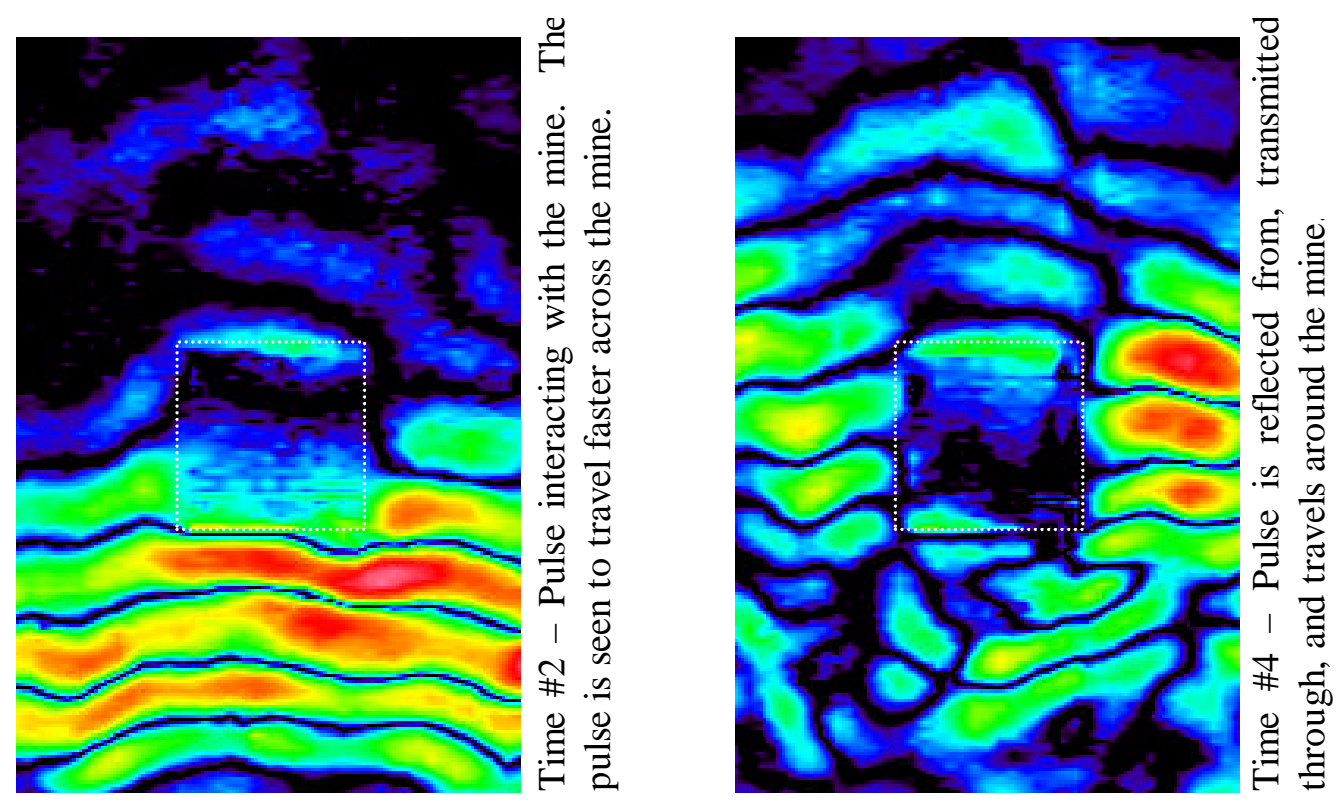

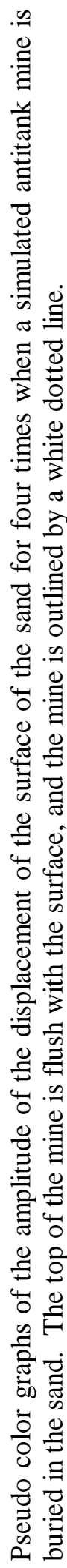
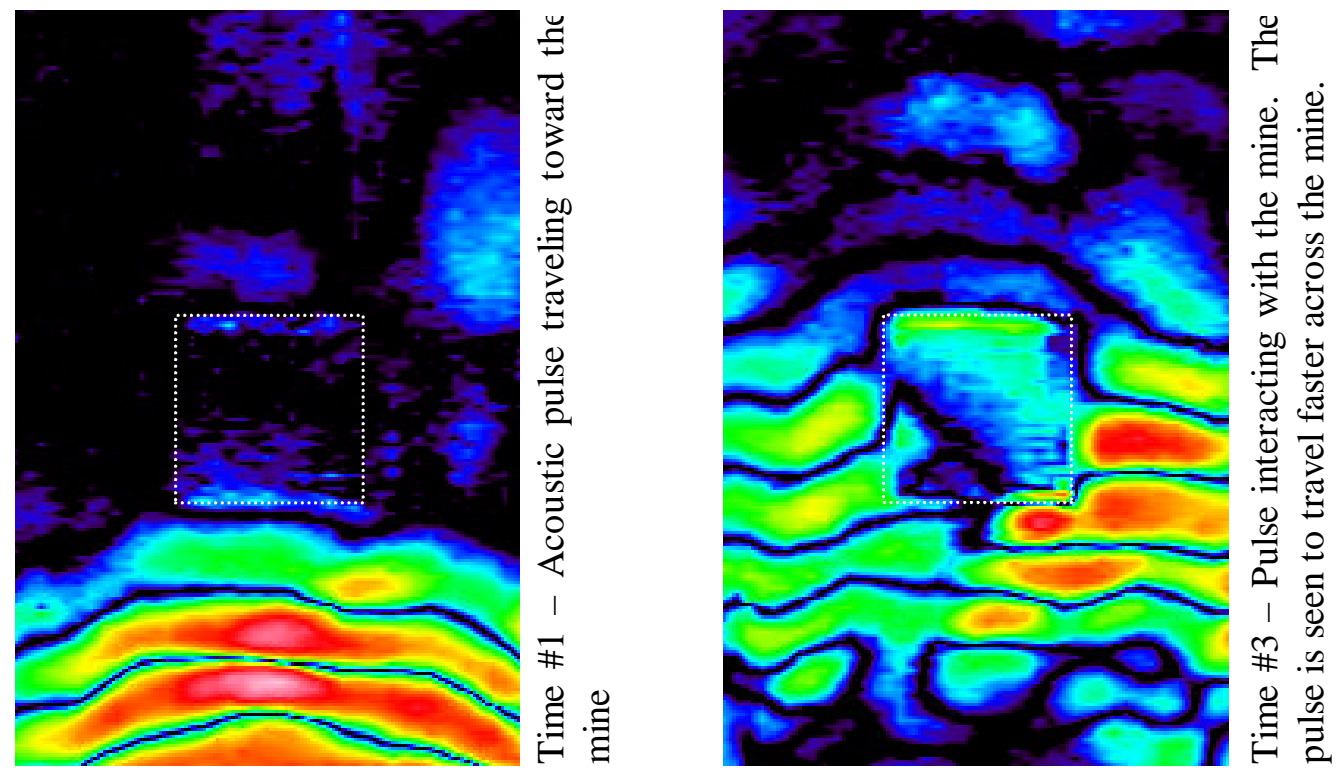

苛 

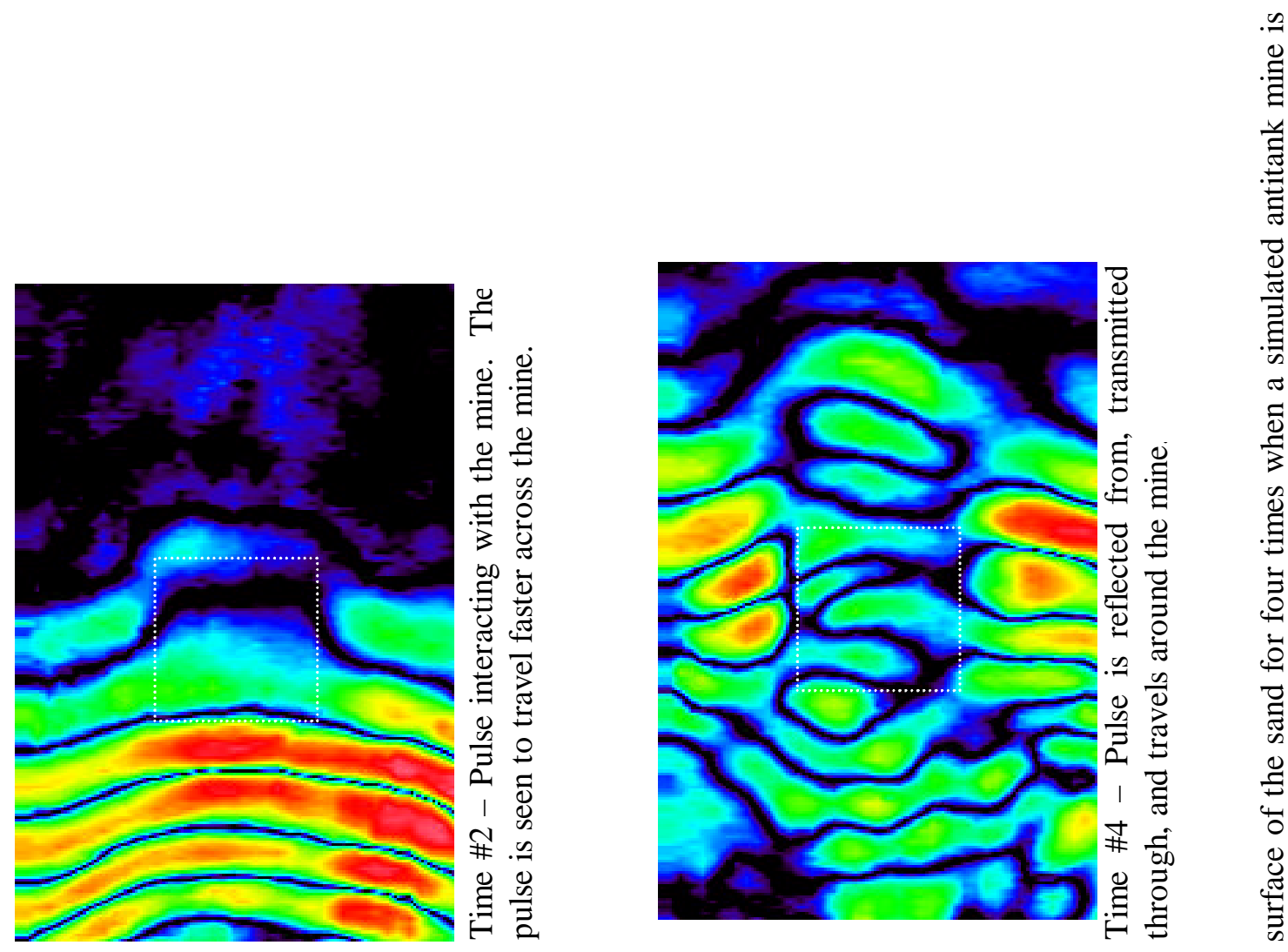

葛

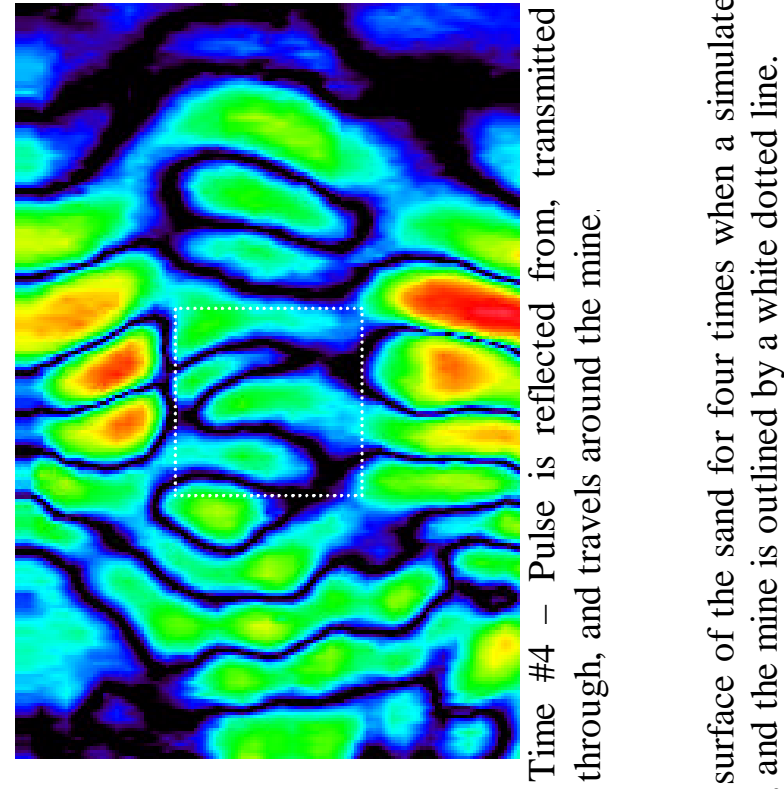

咨

$\sigma$

을

夰

층

으.

芯

喅

ن

흘

¿

渮

홍

ए

$\frac{\pi}{2} \frac{0}{0}$
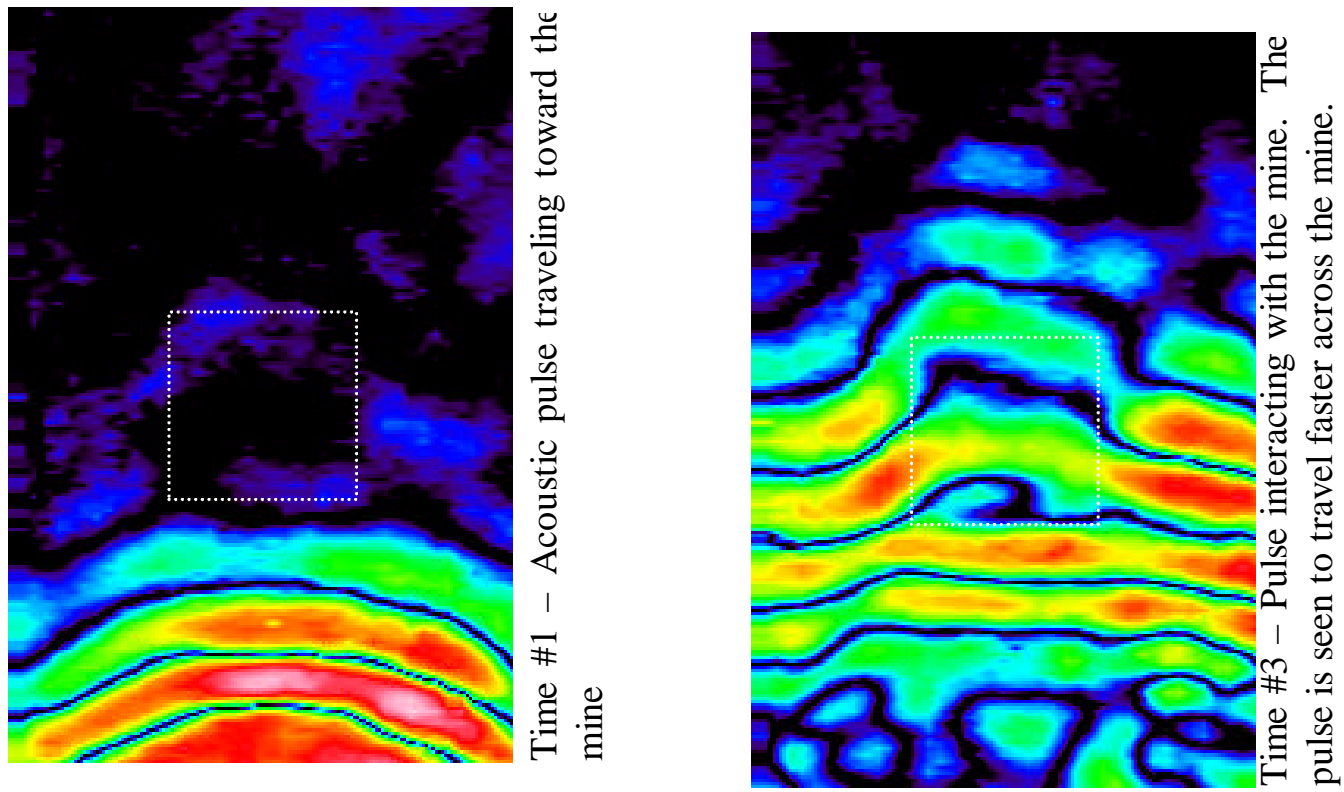

으

的

ㄴㅇㅇ

导.䒜

을

需

ㅇ

응

हैं

응

قِ

吕 


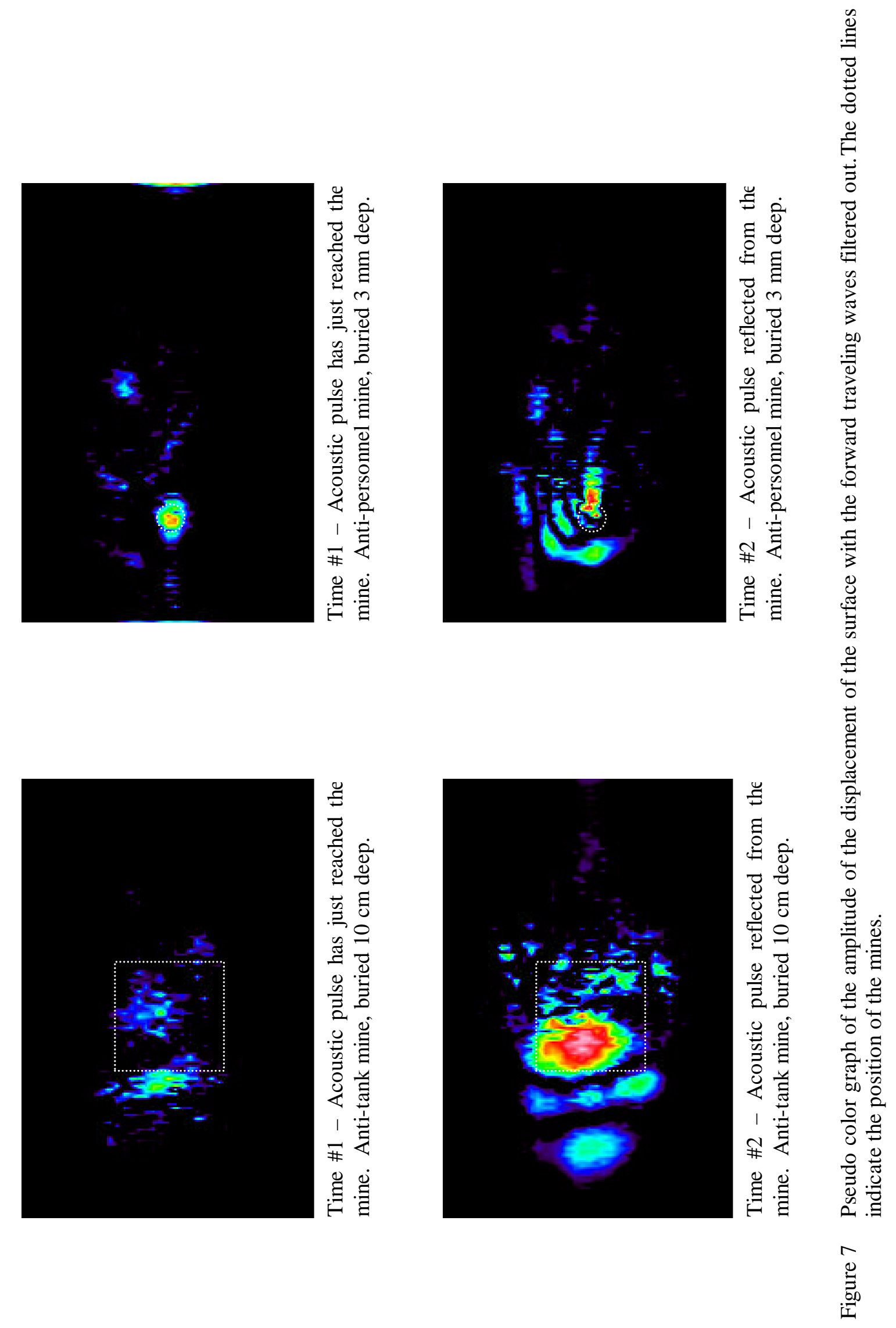




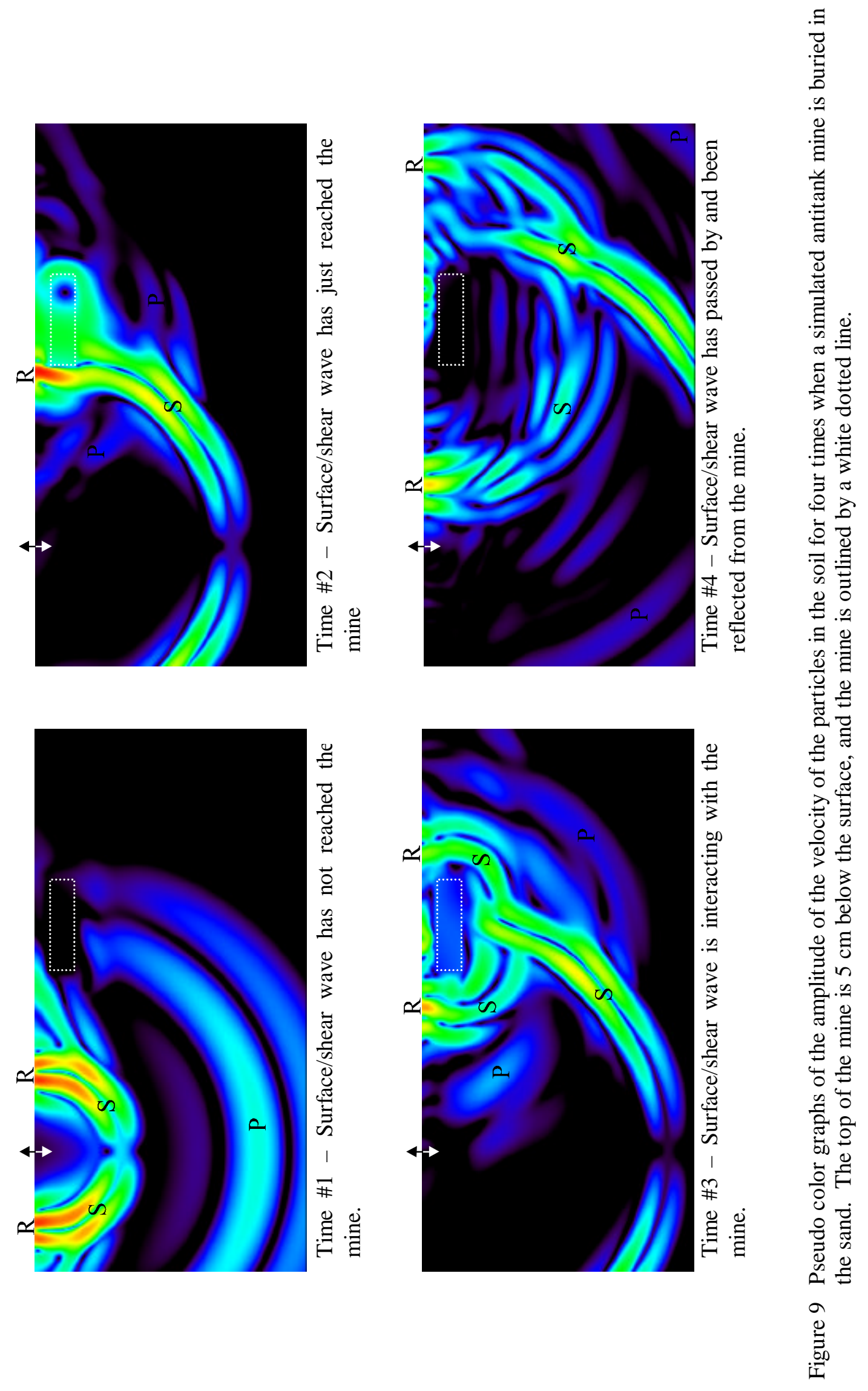

